\title{
Not Just for Ornithologists
}

\author{
Speciation in Birds, by Trevor Price. Greenwood Village, CO: Roberts and Company, \\ 2007. Pp. $x+470$. $S / b \$ 59.95$
}

\author{
Beatrice Kondo
}

Published online: 7 April 2010

(C) Springer Science+Business Media, LLC 2010

Speciation in Birds begins with a justification for using birds to study the subject of speciation. The introduction and the entire volume are successful in this endeavor. As the author points out, birds have been heavily studied in the key works on speciation, from Darwin onward. As discussed in the first chapter, genetic studies of speciation (Dobzhansky 1951; Coyne and Orr 2004) do not include as much research on birds, but that does not detract from the thorough grounding that Speciation in Birds will impart to the general audience, even while it provides original research of interest to the professional evolutionary biologist. The text broadly covers geographic and ecological factors affecting speciation, including an illustration of how they form a spectrum of possible factors, rather than two mutually exclusive possibilities. Then the book expands into social selection (including sexual selection), tying both of these into a geographic and ecological framework, and finishes with a discussion of hybrids, hybrids zones, and genetic incompatibility.

Throughout, the text scrupulously avoids the use of jargon and gives clear definitions of terms, as well as uses concrete examples to illustrate key concepts. Thus, the reader learns the terminology without having it hamper the flow of the reading. For example, the explanation of the phylogenetic species concept (pp. 6-8) includes a clear statement of criteria, examples of usage, and a discussion of confounding factors. The reader may not have been aware that any other definition of "species" than the biological species concept existed, but after the introduction, the ongoing debate on species

\section{B. Kondo $(\square)$}

Department of Biology, The Johns Hopkins University,

3400 N. Charles St.,

Baltimore, MD 21218, USA

e-mail: bkondo@jhu.edu definition becomes clear. Without choosing between species concepts, the author succeeds in getting past the hurdle of how species are defined, to the much more important question: their origin. While the academic debate about the dozens of species concepts will undoubtedly continue (although they are perhaps winding down [Hey 2006]), the author focuses on the historical processes causing speciation, rather than arcane points about how to define a species. He states succinctly: "Without reproductive isolation, only one species would be found at each location in the world" (p. 8). Note that this is not an attempt to use the biological species concept as a criterion for determining species status (see Mayr 1982), but rather a statement about origin of species. Two other topics that are technically complex (and the subjects of continuing research and refinement) are discussed as appendices.

Chapter 2 contains two additional sections after the summary-Appendix 2.1: Molecular Dating Methods and Appendix 2.2: Phylogeny Estimation. These appendices can be skipped with no loss of continuity, but provide the basic framework for understanding molecular clocks (2.1) and describe the author's methods of estimating phylogenetic relationships. The molecular clock appendix carefully discusses relevant technical challenges, theoretical models, and assumptions underlying the use of these techniques. The descriptions are clear, and the author describes the caveats associated with his own choices in implementing these methods throughout the remainder of the book. Thus he states that for evolutionary times over one million years, he uses a molecular clock of $2 \%$ per million years, but the reader understands why this is an approximation, what factors affect the accuracy of the estimate, and why it is nevertheless a useful rate for the purposes of the text. The appendix on phylogeny estimation discusses the author's methods and explains the use of mitochondrial DNA for examining closely related species. What is omitted in this 
appendix is a discussion of the pitfalls in using only one gene locus: selective sweeps, violations of neutrality, and the general idea that with only one locus, what is being estimated is a gene tree, not a species tree. Other than that, this appendix is an excellent introduction to some basic methods in phylogenetic estimation.

This text could serve as a good upper-level undergraduate or graduate textbook, as well as provide original research that should be of interest to systematists and evolutionary biologists (for example, the comparison of island size to community composition, pp. 54-56). Those completely unfamiliar with speciation and natural selection can expect to be intellectually challenged by the reading, simply because there is so much material (over 400 pages) and thus not much repetition to re-establish a theme or framework after each new concept. The author sets up the framework in the introduction, and thereafter it is up to the reader to keep track of how the rest of the book fits into that framework. Nevertheless, each chapter is organized into sections with meaningful headings and ends with a conclusion and summary.

What is particularly useful to the reader is the way the author integrates different factors affecting speciation. Each factor, such as ecological differences or geographic separation, is defined and discussed with examples that illustrate the mechanics of how speciation occurs because of that factor. Then discussion proceeds to an examination of how factors interact. For example, in discussing geographic isolation and ecological differences, the author presents a graph of speciation probability (Fig. 2.3, p.17), involving both geographic separation and ecological differences. Ultimately social and sexual selection, as well as barriers against hybridization once speciation has commenced, are woven into the tapestry of speciation.

One particularly unusual method of examining speciation in this text is the comparison of variation in domestic species with variation in related wild species, for example, domestic Rock Dove breeds and wild doves and pigeons (Table 11.3, p.229). Examining domestic breeds' rapid divergence from each other as well as from the wild ancestor gave Darwin many insights about the process of speciation (Darwin
1871). Although reproductive success is determined by the breeder, and selection on most traits is much more relaxed than in the natural setting, artificial selection has striking parallels with sexual selection, particularly in situations of low predation or other environmental stresses. The traits that diversify with greatest frequency between different breeds of the same species (Table 11.1) are traits that diversify under sexual selection in nature. The implications of single-gene and polygenic mutations, as well as the higher prevalence of breed-distinguishing mutations on the $\mathrm{Z}$ chromosome (the non-degenerate sex chromosome in birds) are also discussed. Finally, the author notes the tendency, under natural selection, for parallel evolution, which is less common in artificial selection due to relaxation of selection on survival traits in domestic species. Overall, this chapter makes a strong argument to downplay the role of runaway selection in the sexual selection process, making good use of the parallels between artificial selection and natural selection.

Speciation in Birds provides illustrative examples and quantitative analysis of the major players in speciation theory and species concepts. Educators will find that it provides concrete understanding of basic theory, while also delving into nuances that are the subject of thriving debate and ongoing research. The text as a whole is a rich tapestry of the field and could form the framework for an advanced class in this area; likewise, it would be an excellent reference for use in journal clubs or seminar classes.

\section{References}

Coyne JA, Orr HA. Speciation. Sunderland, MA: Sinauer; 2004.

Darwin C. The descent of man, and selection in relation to sex, vol. 2 . London: John Murray; 1871.

Dobzhansky T. Genetics and the origin of species. 3rd ed. New York: Columbia University Press; 1951.

Hey J. On the failure of modern species concepts. Trends Ecol Evol. 2006;21:447-50.

Mayr E. The growth of biological thought: diversity, evolution, and inheritance. Cambridge, MA: Belknap Press of Harvard University Press; 1982. p. 273. 